\title{
Visual Abstraction of Complex Motion Patterns
}

\author{
Halldór Janetzko ${ }^{a}$, Dominik Jäckle ${ }^{a}$, \\ Oliver Deussen ${ }^{a}$, and Daniel A. Keim ${ }^{a}$ \\ ${ }^{a}$ All authors are with University of Konstanz. \\ E-mail: firstname.lastnameduni-konstanz.de.
}
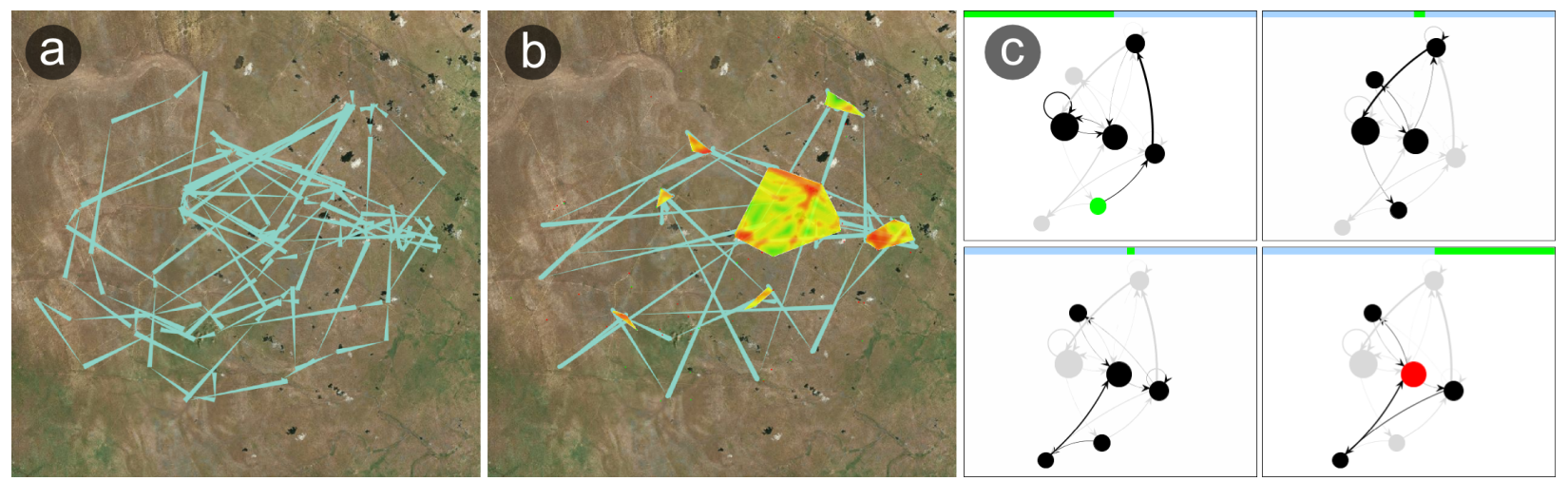

Figure 1. In order to reveal hidden information and patterns in motion data (a), we propose two sequential techniques: the geospatial (b) and the temporal abstraction (c). The geospatial abstraction uses a density-based approach to cluster very dense areas based on user chosen properties. The temporal abstraction segments and visualizes the results of the geospatial abstraction as a graph network, represented in several small multiples. Through an interactive feedback loop the user is able to explore and analyze the trajectory.

\begin{abstract}
Today's tracking devices allow high spatial and temporal resolutions and due to their decreasing size also an ever increasing number of application scenarios. However, understanding motion over time is quite difficult as soon as the resulting trajectories are getting complex. Simply plotting the data may obscure important patterns since trajectories over long time periods often include many revisits of the same place which creates a high degree of over-plotting. Furthermore, important details are often hidden due to a combination of large-scale transitions with local and small-scale movement patterns. We present a visualization and abstraction technique for such complex motion data. By analyzing the motion patterns and displaying them with visual abstraction techniques a synergy of aggregation and simplification is reached. The capabilities of the method are shown in real-world applications for tracked animals and discussed with experts from biology. Our proposed abstraction techniques reduce visual clutter and help analysts to understand the movement patterns that are hidden in raw spatiotemporal data.
\end{abstract}

Keywords: Trajectory visualization, moving objects, abstraction

\section{INTRODUCTION}

The ever-increasing amount of tracking data leads to a high interest in understanding and analyzing movement behavior. Logging and tracking movement using GPS devices or even smart-phones is getting easier and cheaper as technology advances. Furthermore, since smart phones are typically worn by people they can be used to track and at the same time to analyze and to visualize their motion. Services such as Google Latitude already provide end users with the ability to track their motion and to automatically tag locations as work places or homes. Having such small devices at hand, detailed analysis and visualizations methods for geographic motion data are getting more and more important. Geospatial advertisement, for example, is a promising field but yet still not really supported by most systems and further research is needed.

The main challenge is to deal with the large amount of data with high resolution and covering long periods of time. Typically, such trajectories include a high degree of over-plotting. Humans have their daily travel to work and animals 
have sleeping and foraging areas. Territorial animals such as wolves or lions stay in their territory and visit other places only in rare and exceptional cases. Migrating animals have a different behavior and might also have areas where they stay pretty long for breeding or hibernation but also travel long distances. Movement on such different scales is quite difficult to visualize without using distortion techniques as small movement patterns will be over-plotted or the global context is lost. In this paper, we focus on animal movement as privacy in human generated data is often an issue. Nevertheless, our technique is applicable to all kinds of movement data.

The relationship between such different kinds of motion is the following observation: regions with a high density of visits are connected by low-density transition movements. For humans and migrating animals this observation holds obviously true. In the case of territorial animals it is more complicated. The overall geospatial density distribution of points does not help much when analyzing such behavior. However, as soon as we take the time into account and look for motion changes over time we see patterns that are invisible before. Figure 2 shows an example of such temporal shift in the movement of a lion. The colormap represents density values and uses green for low densities and goes over yellow to red to encode high densities. The visualization of the whole trajectory in Figures 2(a) and 2(b) does not reveal the hidden temporal pattern. By partitioning the trajectory into three parts in time the northern trajectory of the first third (Figure 2(c)) and the north-south transition in the last third (Figure 2(e) becomes visible. Only by visualizing different time slices the analyst is able to observe such patterns, so the crucial point is how to determine and to find meaningful time slices?

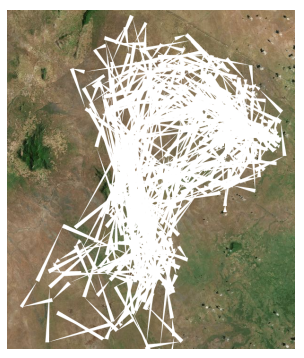

(a) complete trajectory

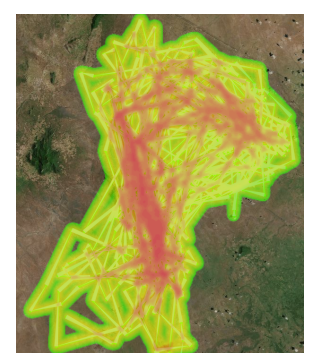

(b) density visualization (c)

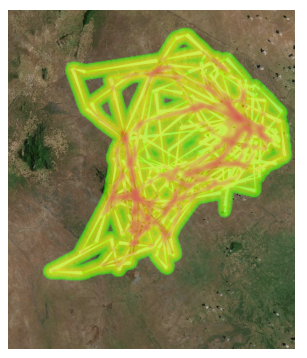

tory

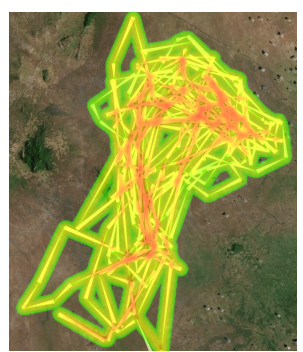

(d) second third of trajectory

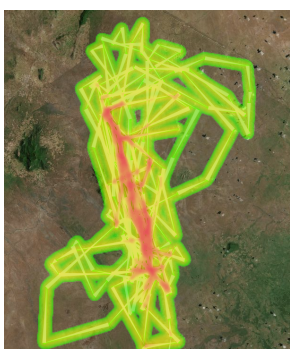

(e) last third of trajectory

Figure 2. Territorial motion data of a lion over a period of two years. The figures (c) to (e) show the temporal shift of the geospatial pattern which is not visible in the complete trajectory shown in (a) and (b). The density visualizations encode low density areas with green, medium density with yellow and high density with red colors.

In this paper we present an approach to automatically detect dense regions and use them for visual abstraction of complex motion patterns. The above-described observation is the guideline for our technique. We partition the trajectory into regions with high density and transitions between them. Additionally we analyze and detect temporal shifts in movement patterns and use them for what we call temporal abstraction. The contributions presented in this paper are three-fold:

1. user-controlled density-based analysis of trajectories

2. geospatial aggregation and simplification for visualizing transitions between regions of interest

3. temporal partitioning of trajectories according to movement changes

Detailed explanations for our proposed methods are given in Section 3 after sketching related work where we summarize state-of-the-art techniques with respect to their methodological background. Our technique is applied to real-world trajectory data of animals in Section 4.2. We evaluate the proposed method using expert feedback and finally conclude our work with an outlook on possible future works.

\section{RELATED WORK}

Many techniques for visualizing and analyzing geospatial movement data have been proposed in the past. Their goal is to enable analysts to detect and understand movement patterns in large datasets. As mentioned above, the visualization of 
dense data sets is quite challenging. Simple statistical methods like sampling, for example, will not work for the dataset shown in Figure 2(a) because the amount of over-plotting is by far too high. Three methodological approaches are dealing with such complex trajectories. The first one is to remove unimportant parts of the data by using simplification approaches. The second one is aggregation. Here data is collected in bins and only common properties for each bin are shown. A third approach works with distortions. Since it affects geospatial topology it is not discussed here. The following two subsections will present techniques for the first two approaches. We also describe methods for segmenting trajectories into coherent parts, an idea closely related to our approach.

\subsection{Simplification-based approaches}

Line simplification is important for drawing readable maps. An example are coastal lines on different scales. A popular, purely geometric line simplification approach is the Douglas-Peucker algorithm ${ }^{[1]}$ It recursively retains all significant points and removes insignificant ones. The significance of a point is computed by its distance from an approximating polygon line that is determined recursively. Other geometric approaches for simplification are presented in a work by Nake et al. ${ }^{[2]}$ Smoothing techniques for different line types such as angular lines, sinuous lines, and contour lines are presented by Burghardt et al ${ }^{3}$ Agarwal et al ${ }^{[4}$ propose an algorithm for computing simplified curves for geographic applications. Another class of simplification methods reduce the temporal resolution of a trajectory. The influence of changes in temporal sampling towards derived attributes like speed and sinuosity is studied by Laube and Purves. $\frac{5}{5}$ The technical background of sampling methods for visualizations is presented by Ellis et al. ${ }^{6}$

Only to a certain extent it is possible to apply techniques for efficient map rendering to movement data and trajectories. Coastal lines and buildings, for example, do not inhibit self-crossings or over-plotting and therefore typically do not fit to geospatial movement. The Douglas-Peucker algorithm is applicable to general trajectories but the resulting curves may not express important and unknown patterns. Since the result is mainly based on outliers in dependency to straight line approximations it was not very helpful (see Figure 2) for our trajectories.

\subsection{Aggregation-based approaches}

Aggregation-based methods avoid over-plotting at all and furthermore allow also a large amount of complex trajectories. There are several techniques applying aggregation to movement data. Density visualizations using surfaces represent often visited regions by heatmaps. ${ }^{7.12}$ Flow maps group similar movements together by partitioning the spatio-temporal space and visualize movements as a directed graph ${ }^{[13+16}$ Spatially ordered treemaps introduced by Wood et al ${ }^{[17}$ can be used to display trajectories by aggregation. Methods for spatio-temporal aggregation are proposed by Andrienkos, $\frac{18}{18}$ an overview of existing approaches can be found in the framework by Andrienko et al ${ }^{19}$ Another very related application area is the visual analysis of eye tracking data. Andrienko et al. ${ }^{[2]}$ and Li et al. ${ }^{21}$ present different approaches based on clustering and the space-time cube. More related to the area of animal movement data also using aggregation-based techniques is the work of Grundy et al. ${ }^{22]}$ In this paper, we combine the partitioning of trajectories with density visualization and furthermore provide a flow-based graph representation.

\subsection{Segmentation of Trajectories}

For representing trajectories in an understandable and meaningful way is very helpful to segment the movement into coherent parts. Doing so we can visualize trajectories in a more expressive way. Segmentation of trajectories into episodes is an ongoing research topic for gaining efficient algorithms. Movement episodes are defined as those parts of a trajectory that have relatively coherent properties such as speed, heading, sinuosity, etc. and that are cut by sudden changes in such properties. ${ }^{[7}$ A new episode starts after a break and ends at the next cut position. A number of spatio-temporal cut criteria are discussed by Buchin et al ${ }^{23}$ Concerning performance, a variety of methods implementing such criteria have been proposed $[24 \sqrt[27]{ }$ We use a slightly modified approach for segmentation as we are interested in regions where the movement of animals fulfills specific properties that label them as sleeping places or foraging areas.

The results of the segmentation process can be used for further analysis and visual presentation. Similar episodes of moving objects are detected and grouped by Laube et al. ${ }^{28}$ The authors look for specific motion patterns of several different moving objects. Motion modes are automatically classified by Dodge et al.29 by performing an analysis of motion characteristics within trajectory episodes. 
The presented methods segment trajectories into coherent episodes with respect to given properties. We integrate density-distributions into such segmentation processes. Our approach is related to these techniques as we also partition trajectories into points which belong or not belong to interesting regions based on user-chosen properties.

\section{TECHNIQUE}

Our approach is to first look for data properties allowing to partition and aggregate the trajectories in a meaningful fashion. As trajectories use temporal and the geospatial dimension, we will describe abstraction methods for both. First, we will explain in detail our approach for the visual abstraction in the geospatial dimension. The temporal dimension is the focus of the second part of this chapter and deals with the automatic detection of coherent time windows. A short movie presenting our approach can be found at http://files.dbvis.de/files/atravi.mp4.

\subsection{Geospatial Abstraction}

The basic idea of our technique is to visually abstract the trajectory when certain properties are fulfilled (e.g., low speed or bad weather conditions) and to show only simplified transitions. More specifically, we reduce the amount of overplotting by a process of filtering, clustering, and finally visual abstraction. The overall abstraction process is depicted in the schematic Figure 3 In the filtering step (I), the analyst can at first interactively select points of the trajectory with specific attribute properties. We therefore provide filter functionality for attributes like speed, heading, or duration and also for additional attributes like weather information as precipitation.

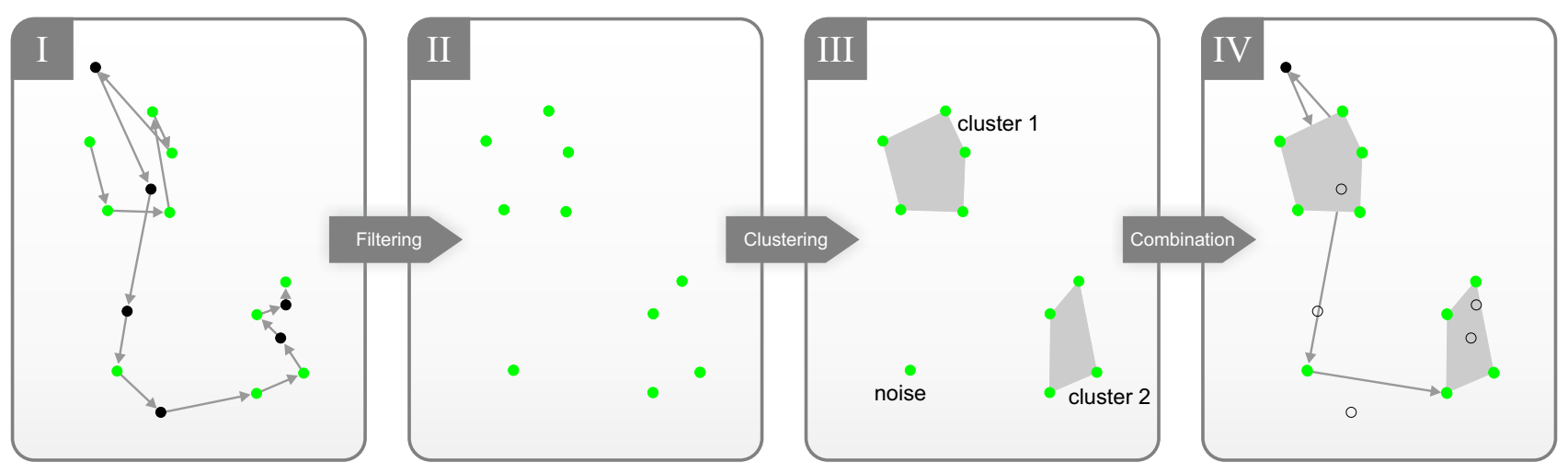

Figure 3. Process pipeline of the visual abstraction beginning with the raw data in (I) applying filtering (II), clustering (III), and finally combining and visualizing the results in (IV). Only the green points fulfill the user selected filter criteria.

The user interface for filtering is capable of a concatenation of several filters tailored to the analyst's needs. For a more effective filtering we support the analyst by providing a histogram of the attribute's value distribution. All data points passing the filters, shown in Figure 3 (II) are then clustered by DBSCAN, 30 a density based clustering technique, providing the result in Figure 3(III). The user can control the clustering granularity as he can influence the epsilon and MinPoints parameter interactively. For each cluster we then compute the convex hull and use it as an visual abstraction for this part of the trajectory. In addition to drawing the convex hull polygon, we fill each convex hull with the density distribution visualized by a heatmap. The colormap depicted in Figure 2 encoding the density distribution goes from green (low density) over yellow (mid density) to red (high density). The last step is to visualize the trajectory on a geographic map, while using the previously computed convex hulls and using simplified trajectories in between. For simplification purposes we use only one intermediate point between the clusters. Simplifying the transition trajectories can be performed by applying the Douglas-Peucker simplification for example. We iterate over all points of the trajectory and look only for points being the transition from one convex hull to another. Note that we also handle cases where these two convex hulls are identical and still show the transition. These transitions are then visualized in a simplified version to only give a rough overview of the transition course, depicted in Figure 3 (IV). A final result of the transformation process can be seen in Figure 4, where we applied our method to a trajectory of an albatross. 


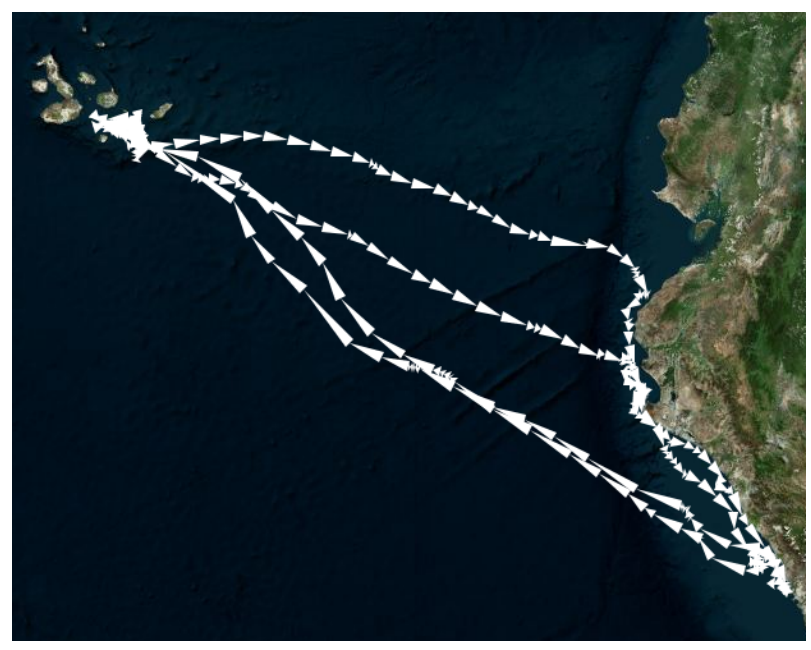

(a) original trajectory

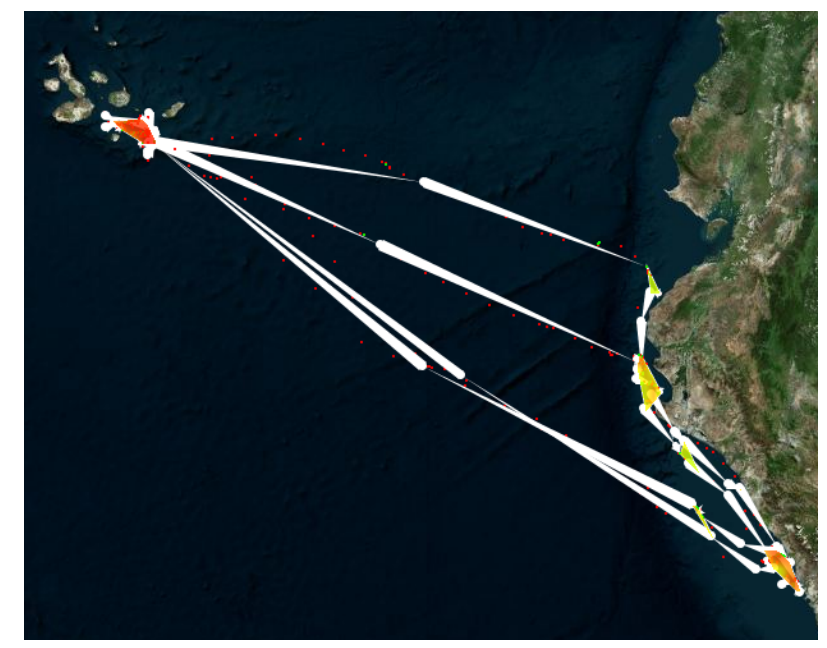

(b) geospatial abstraction

Figure 4. Our geospatial abstraction method is applied to an albatross trajectory presented on the left hand side. The final result of the proposed method is shown on the right side. We used for filtering a low speed in order to find resting and foraging places.

We do also provide another visual representation of the processed trajectory. Basically, the convex hulls and the transitions in between can be seen as a graph network. We therefore implemented a graph visualization of the trajectory with the convex hulls being the nodes and the transitions being the directed edges. The graph layout is implemented in a way that it reflects the geographic relations while providing a very high-level and abstract overview of the data. The graphbased and the map-based representation can be shown side by side supporting Brushing and Linking. The interaction between both techniques gives the analyst an overview graph, showing the major moving patterns, and enables further detailed analyses in the map-based visualization.

\subsection{Temporal Abstraction}

The result of the geospatial abstraction described in the previous section 3.1 is used as an input to our temporal abstraction algorithm. We visualize the identified convex hulls and transitions as a graph network. The graph network is an abstract representation of the processed trajectory, but does per se not include additional information compared to the map visualization. Therefore, we add supplemental information to the graph network to enable further analysis steps. Each identified cluster as well as all transitions between these clusters contain several points holding spatio-temporal information. We are using both, the time and the spatial location of these points, in order to enhance the visual representation for deeper understanding of the movement data.

Figure 5 gives an overview of the overall transformation process. At first, we need to map the position of a cluster, visualized by the convex hull, to the node position in the graph network. We want to keep the displays as similar as possible and retain the relative positioning as best as possible. The convex hull consists of multiple points and we therefore calculate the centroid of the convex hull defining the position of the node. This step visually aligns the representation on the map with the abstract representation as graph. Once the position has been calculated, several different attributes can be used to affect the node size: The amount of points enclosed by the convex hull can give a global overview in which cluster most of the time was spent. But the size can also be determined by mapping the size the enclosed area to the node size. Another possibility is to map the time span a cluster was visited to the node size. The application of these different parameter mappings depends on the analysis.

The transition from one cluster to another is visualized by a directed edge. In order to reduce visual clutter, multiple transitions between the same two clusters are aggregated and handled as one. If an moving object, for example, often leaves the convex hull and returns immediately, we represent this as one single reflexive directed edge. Here also the possibility is given to use different attributes to affect the stroke width. The user has two possibilities: Either using the amount of intermediate points or the amount of transition revisits. 


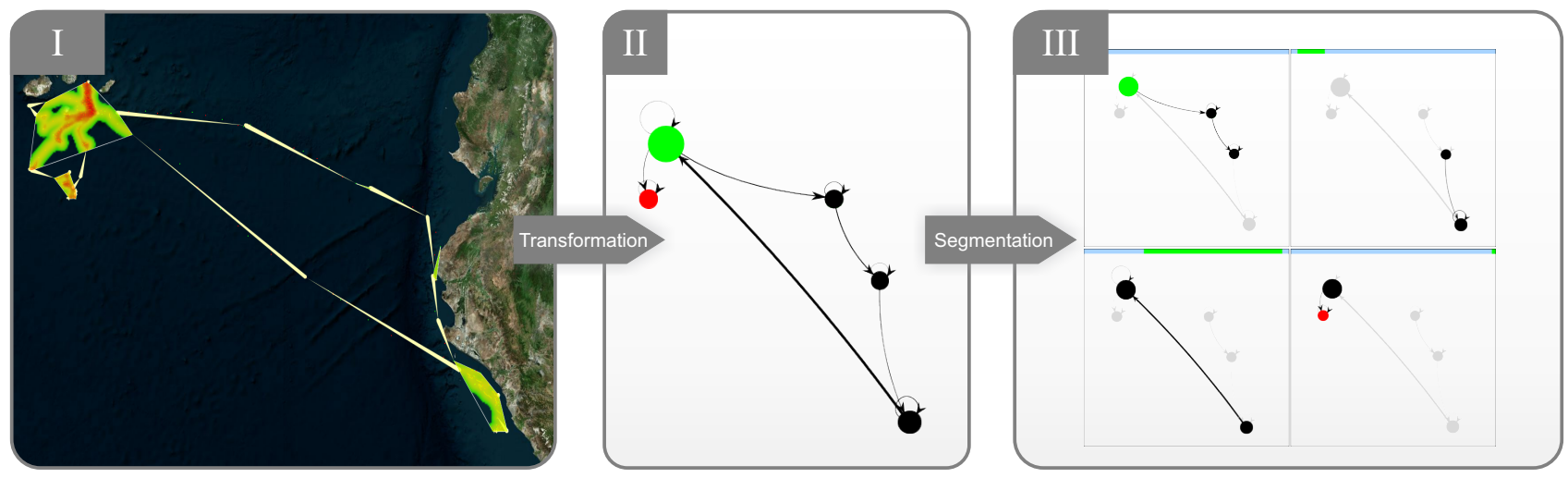

Figure 5. Exemplified abstraction of the map visualization (I) into a graph network (III). All clusters are represented as nodes and transitions as directed edges. The position of the nodes of the graph (II) corresponds to the relative geospatial positions of the clusters preserving a visual alignment of map and graph. The resulting graph is partitioned into several graphs in (III), each representing a certain timespan and a certain number of transitions.

Crucial for the temporal abstraction is the handling time in a meaningful and expressive way. Our goal is to retrieve further information like motion patterns and therefore a segmentation of the motions according to different criteria is needed. One possibility is, to divide the motion data into several equal-time intervals and to visualize the visited nodes and edges. In this case, at least two severe problems occur: First, selecting a fixed time span may lead to patterns not being visible as they might cross the border between the time spans. Second, by drawing the affected nodes and edges only the context of the motion pattern is missing. It may be impossible to put the affected nodes and edges into context with respect to the entire graph network. The less nodes a motion pattern covers and the bigger the entire graph is, it is more and more difficult to visually map the nodes. Hence, we propose our technique combining the overview and detail methodology $\sqrt{31}$ with an semi-automatic algorithm for defining the time intervals dynamically. Furthermore, we integrate the small multiples technique ${ }^{32}$ to visualize all time intervals side-by-side. Small multiples are the combination of several snapshots of the same visualization in a dashboard-like representation; in our case, every snapshot represents a different time window. Furthermore, we enable the analyst to influence the time segmentation process according to his needs.

As stated above, the geospatial context of the motion pattern may not be retrievable when visualized alone. As a context visualization we draw the entire, fully connected graph, but gray out all nodes and edges not being contained in the calculated time span. In addition, the very first node of the trajectory is drawn green and the very last node is shown in red.

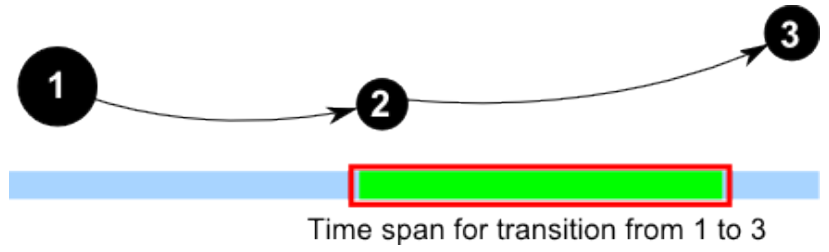

Figure 6. This figure shows the determination of the time span. The time span starts when leaving cluster 1 and ends when entering cluster 3. Notice, that the points contained by the clusters 1 and 3 do not contribute to the identified time span.

As already mentioned, the time intervals are calculated automatically and do not cover equal long time spans. Algorithm 1 shows the computation process in more detail. We try to avoid to partition the graph at points in time where the motion pattern would be interrupted, for example in the middle of an intermediate edge between two clusters, we propose the usage of a threshold. This threshold decides after how many hops the motion will be interrupted to create a new small multiple or rather to end the time interval and to start a new one. The user can change the threshold interactively and directly influence the partitioning process. Figure 5 (III) shows the result for the threshold zero. Every transition from one cluster to another is visualized in a separate small multiple.

The segmented graph, resulting from the user defined threshold, covers a certain time span. We determine the exact time span using the temporal information of the aggregated trajectory points. We consequently calculate the temporal 


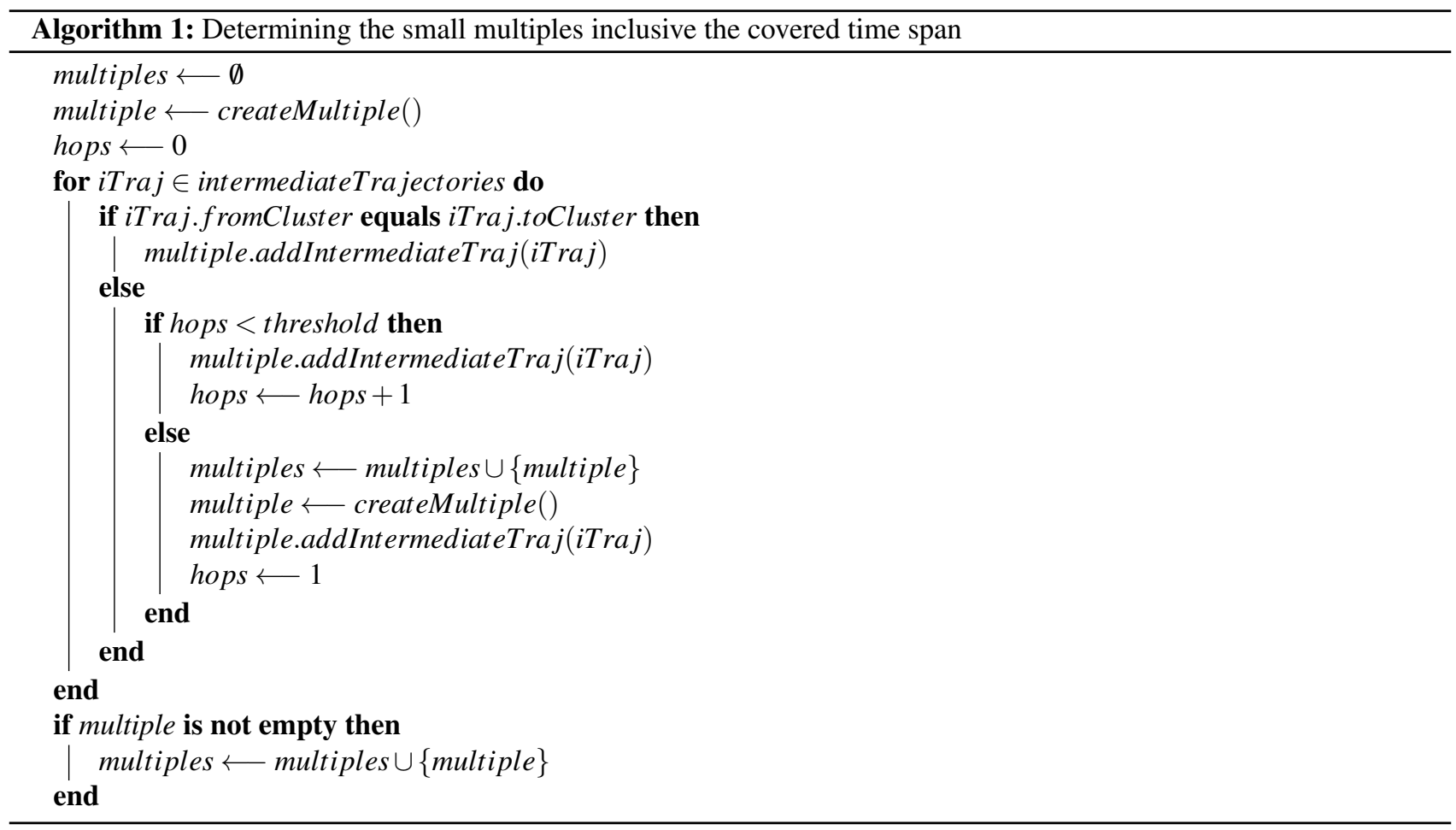

distance between the first point leaving a cluster and the last point before entering the last cluster of the segmented graph (see Figure 6). The so computed time span specifies the covered travel distance. A time bar at the top of each small multiple visualizes the covered time span. The green bar specifies the covered time span in relation to the full span (light blue). The transition between one small multiple to another results in a small gap between the covered time spans. This gap represents the time an mover remains in a cluster before it starts a new journey.

\section{APPLICATION}

For all techniques it is challenging to be applicable for the wide variety of different motion patterns. There are on the one hand very condensed, territorial trajectories and on the other hand large-scale movements with highly varying velocities. We will show that our methods are applicable to every type of movement data. We will apply our techniques to different kinds of animal movement with varying density and velocity properties. More in detail, we will show and analyze movement data of albatrosses and lions with our methods. An albatross travels over long distances between regions with slow movement, whereas lions stays at a certain region and moves inside his territory. In both applications, the amount of points enclosed by the convex hulls is mapped to the corresponding nodes and the amount of intermediate points is mapped to the stroke width.

\subsection{Motion analysis of albatrosses}

The movement data of albatrosses is characterized by varying distances, densities, and velocities. To apply our proposed methods, we analyze an albatross whose movement is between the Galapagos islands and the coast of Ecuador. The motion data of the albatross consists of 1113 points. The covered time span lasts from May 31 $1^{\text {st }}, 2008$ until August $9^{\text {th }}$, 2008. First of all, we apply a filter of the speed determining the computed clusters. Filtering the low speed reflects resting and foraging places. Second, we adjust the parameters for the density based clustering algorithm (DBSCAN). We therefor choose a high amount of minimum points and a low distance rate to determine the clusters. For the graph segmentation we apply a threshold of seven transitions. Figure 7 shows the resulting graphs. 


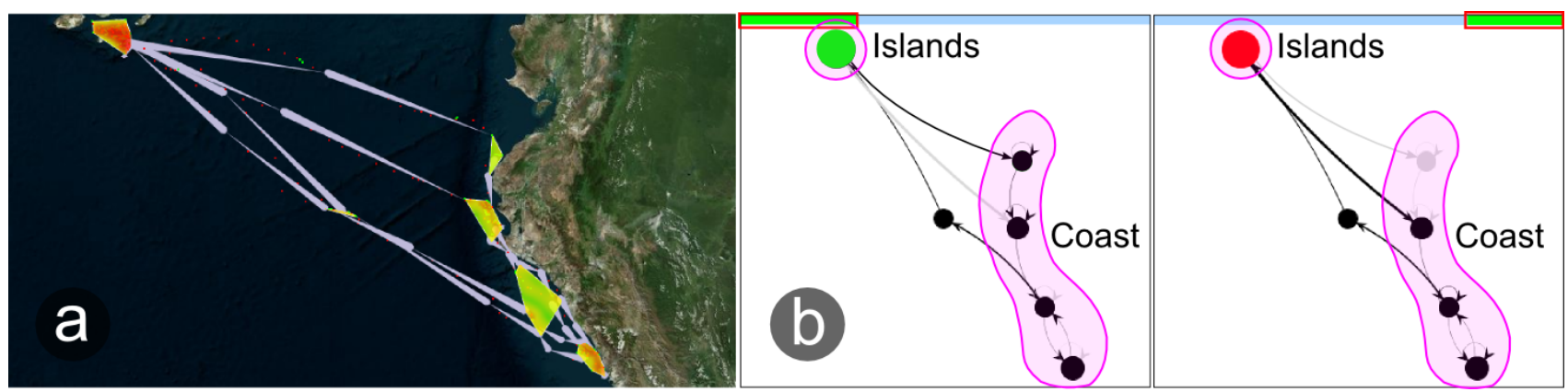

Figure 7. This figure shows the (a) geospatial as well as the (b) temporal abstraction of the movement data of an albatross. The (b) temporal abstraction shows two small multiples with the threshold of seven transitions in order to segment the graph in the biggest cluster (the Galapagos islands) and to unfold patterns. The green, red framed time bar on top of each small multiple visualizes the covered time span.

The threshold of seven transitions has been chosen in order to separate the small multiples at the biggest cluster node. By applying the temporal abstraction, two abnormalities are made visible. The first one is the stopover, represented by the cluster for the Galapagos islands. The chosen segmentation for that cluster unfolds a wide time gap between the two small multiples. This time gap is made visible by the time bar. The time bar for the small multiple on the left hand side visualizes the time span for the transition from the starting cluster to the cluster representing the Galapagos islands, which is also the biggest (Figure 7 (b), the green, red framed time span on the left). The time bar for the small multiple on the right hand side visualizes the time span from the biggest cluster to the cluster where the movement ends (Figure 7(b), the green, red framed time span on the right). By comparing both time spans with each other, there is a wide span missing in between. This gap represents the time spend by the albatross on the Galapagos islands. From this inspection we can suspect the Galapagos islands to be his roost.

Another observation is related to the movement along the Ecuadorian coast. The motion patterns and clusters show, that the albatross remains in the bay areas and furthermore all clusters representing the bay areas all have reflexive transitions indicating the circling of the albatross. For this areas it is likely that the albatross was hunting, indicated by the time spend and the circling behavior. After consulting ornithologists, they approved, that the biggest visible cluster - which represents the Galapagos islands - is the bird's roost and the remaining clusters along the Ecuadorian coast represent the birds hunting grounds.

\subsection{Motion analysis of lions}

Compared to albatrosses, lions do usually not cover wide distances. Hence, the motion data is very dense being challenging for visual analyses. In this section we will compare the motion data of two different lions, living in the savanna. For the first one, Figure 8 shows (b) the geospatial as well as (c) the temporal abstraction of (a) the raw motion data. The motion data consists of 396 points and the covered time span lasts from April $28^{\text {th }}, 2002$ until September $13^{\text {th }}, 2002$.

We choose to filter the speed, so that only the motion with a low speed is being considered. Furthermore, to adapt the number of small multiples to the movement we choose a threshold of three transitions. Figure 8 (b) shows the geospatial abstraction and thus a very dense and large cluster. Via this visualization we can see where the lion probably remained most of the time, but we cannot clearly identify the movements in between the clusters; (c) gives us that information and also reveals a moving behavior of the lion: The purple highlighted node in (c) is drawn black in every small multiple. This means, the lion always returns to this place after visiting at most two other places (indicated by the threshold of three transitions). Moreover, the graph shows a second node with the same size of the purple marked node, which means, that in that area the lion did spend the same amount of time, indicated by the amount of spatial points included in that cluster. In the geospatial abstraction the corresponding cluster has not the same size, but contains a very dense movement.

Figure 9 shows the second lion and its movement analysis. This motion data covers the time span from April $28^{\text {th }}$, 2002 to July $21^{s t}$, 2007. Furthermore, it contains 1465 recorded points and is very dense (see (a)). Therefore, we filter for a very high speed rate to refine the motion data in order to identify hunting scenarios and then apply (b) the geospatial abstraction. This visualization contains several small-sized clusters being barely visible due to the very small clusters. To visualize them in a more suitable way we apply (c) the temporal abstraction with a threshold of two transitions. 

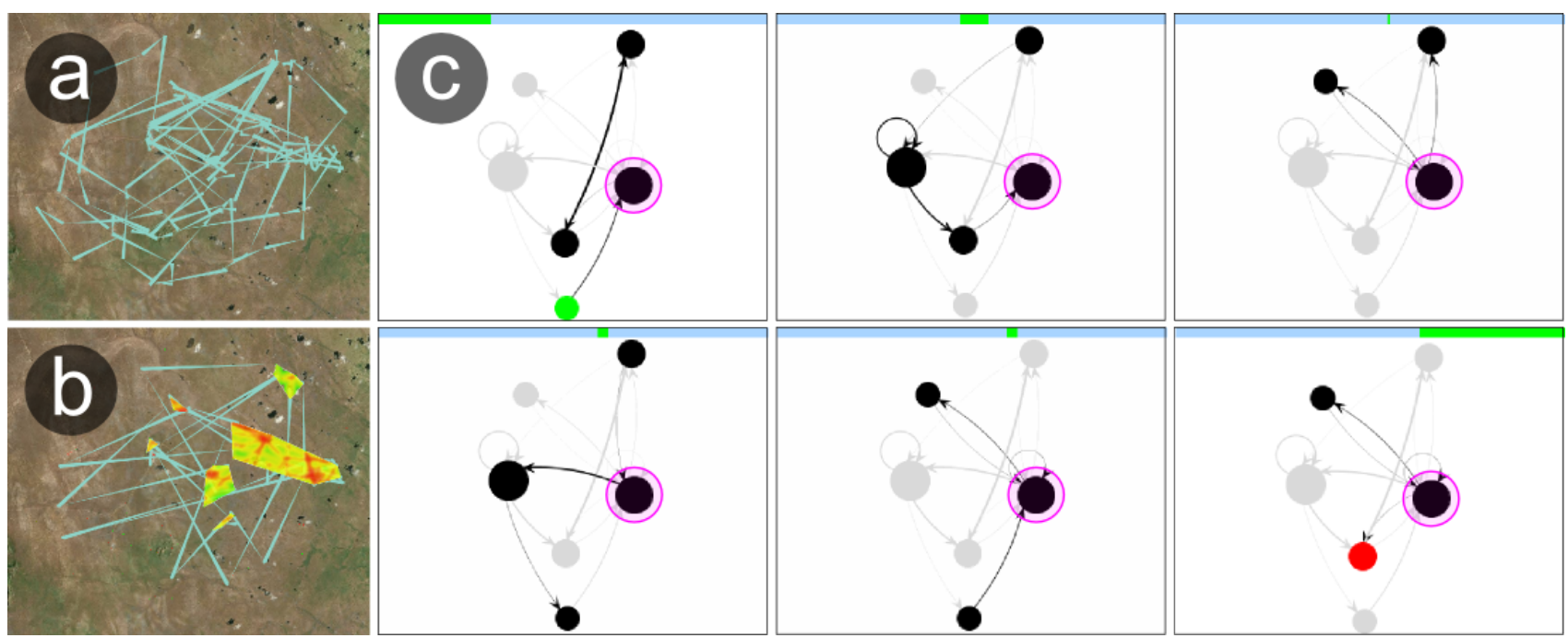

Figure 8. This figure shows the (a) raw movement data, (b) the geospatial, and (c) the temporal abstraction of a lion living in the savanna. Figure (c) reveals the pattern that the lion has one favorite spot.
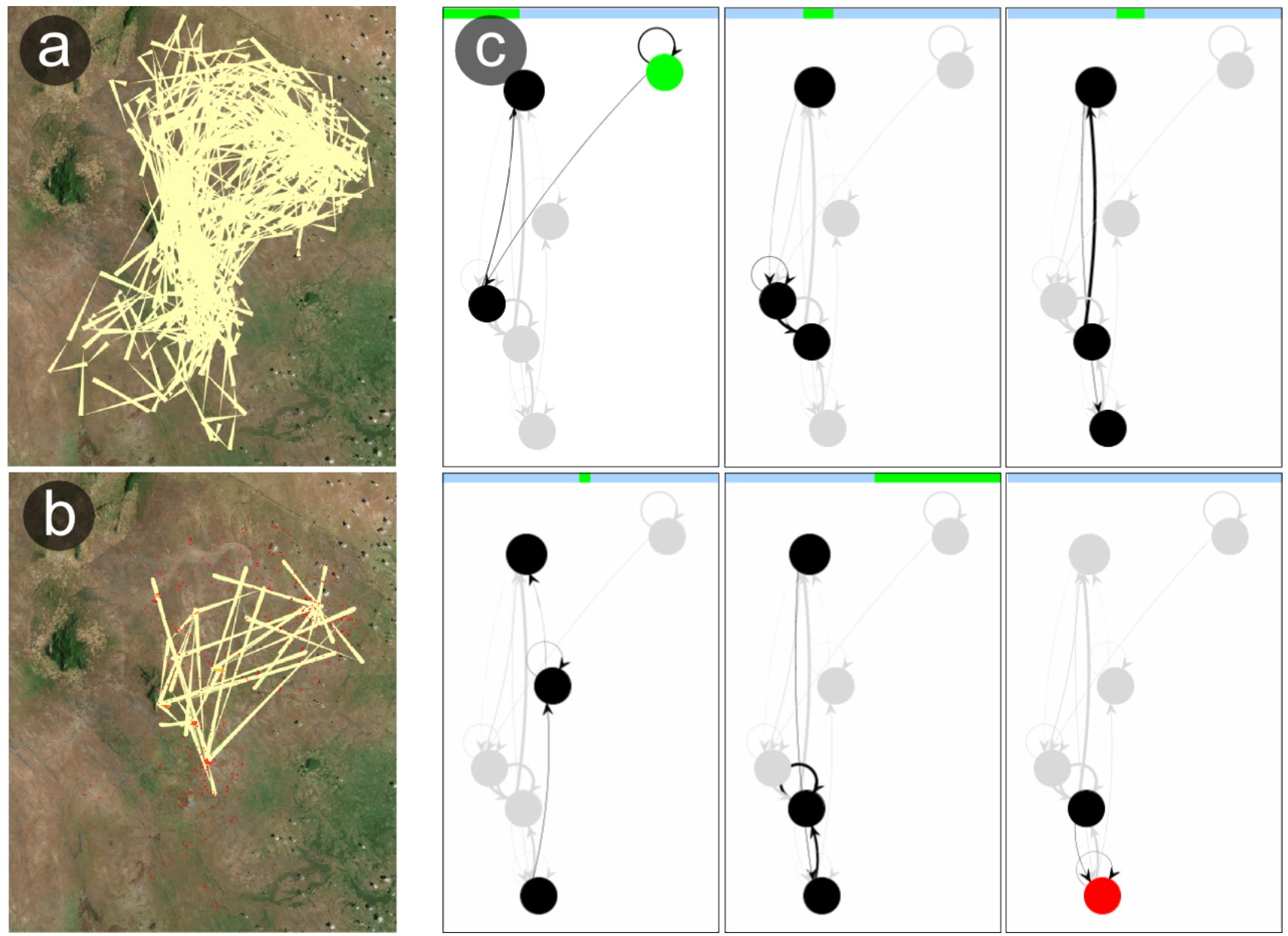

Figure 9. This figure shows the (a) raw movement data, (b) the geospatial, and (c) the temporal abstraction of the second lion living in the savanna as well. Figure (c) shows the abstraction of the hunting grounds. 
The resulting small multiples make these clusters visible to the user. As this motion data has been filtered for movements with a high speed rate, the clusters do stick out, because they highlight a high speed in a dense area. High speed of lions is typically related to hunting and the small multiples reflect the temporal sequence of hunting grounds.

\section{EVALUATION}

Though, we showed the applicability of our proposed technique in the previous application section, it is very important that real users rate our approach effective and helpful. We therefore conducted an expert study with two biologists, both highly experienced in the domain of movement and trajectory analysis. They usually perform their data analyses in commandprocessing analysis environments, like the statistical toolkit R. Interactive parameter setting and immediate visual feedback of our system was therefore highly appreciated. We got very valuable feedback resulting in aspects for future work (see next section) and the following discussions of the merits and limitations of our technique. We first explained our approach to the biologists and afterwards let them interact with our system and look at the different results for the applications described above. We asked them to describe their typical way of analyzing data and furthermore to comment on our proposed technique.

The first feedback we received was related to the two ways of visualizing the same data in a map and in a graph. The biologists stated they would first look on the map for interesting clusters based on the surrounding geographic topology and afterwards investigate the corresponding node in the graph-based representation. They were quite interested in the previous visited node, which was easier to find in the abstract graph than in the map. Furthermore, the directly next visited cluster is also important to them as they want to understand the context of the observed cluster. The identical spatial layout of the graph corresponding to the topology of the map was considered very helpful. The biologists were quite enthusiastic about the fact that they do not have to deal with the high degree of over-plotting anymore. They usually visually inspect only small parts of trajectories due to high degrees of over-plotting or apply aggregation techniques and visualize kernel-density estimations.

Another very interesting result of the feedback session was the usage scenario the biologists described. As described above, our expert users had a high background in statistical analysis, which is quite common for biologists. They typically import their trajectories into $\mathrm{R}$ and perform analysis tasks like statistics or data mining. What they are really lacking is an interactive system enabling them to investigate the parameter space of the applied algorithms. As we showed them our system and let them interactively change the clustering parameters, the biologists were discussing the different clustering results intensively. One outcome was that they would like to have the resulting clusters and polygons in order to import it to R and perform further statistical analysis. Additionally, they would like to have the possibility to annotate the resulting clusters in order to materialize their findings and again export these annotations.

One more serious point arising along our study several time was time importance of the proper filter settings. Only if the analyst knows what he is looking for and is capable of specifying the properties accordingly the analysis process will result in semantically meaningful results. Without any knowledge of the mover's foraging behavior, for example, it will be very difficult to detect these motion patterns. One biologist discussed the difference between migration and foraging patterns for sea birds concerning speed and variation of headings. For migration patterns the heading stays typically the same while traveling quite fast. In the case of foraging, speed is typically lower and variation in motion headings is higher compared to migration settings. In our case, it holds true that the experts know what they are looking for and are able specify filter parameters accordingly. But there might be cases where this is not possible, e.g., looking for unknown behavior patterns like displaying of very rare and shy birds.

What the experts liked from an biologist's point of view is the combination of the home-range philosophy with occurrences of behavioral patterns. The home-range is defined in biology as the region, where the animal spends fifty percents of its time. In our case the home-range can be seen defined by density, because our trajectories are equally sampled. The reason why the biologists liked the combination of both approaches can be seen in one of the higher goals of the biologists. By understanding the resource requirements for distinct motion patterns they can save areas with corresponding resources as nature protection areas and consequently stop the extinction of endangered species. Combining the notion of density with motion patterns has the advantage that areas where the animal showed a specific behavior only once are considered as outliers and not shown in the abstract visualizations. The data basis for further investigations of interesting behaviors is therefore stronger and more convincing when arguing for natural reserves. 


\section{CONCLUSION}

In this paper, we proposed a method enhancing the understanding of complex motion patterns over time for trajectories by applying two visual abstraction techniques: the geospatial and the temporal abstraction. We therefore first performed the geospatial abstraction based on certain user-selected criteria to visually abstract the trajectory and show only simplified transitions. Our presented process pipeline involves, first, a user-driven filter step, second, a density based clustering, and third, the combination and visualization of the results.

We perform the temporal abstraction as second abstraction which uses the results of the geospatial abstraction as input. The identified convex hulls are being visualized as a graph network. Since each cluster contains both, spatial as well as temporal information, we use them to enhance the visual representation. A technique called small multiples is used to visualize the automatic temporal segmentation of the graph. The analyst can influence the segmentation process and adapt the result to his needs. We applied our techniques to two real-world trajectory data sets in the animal domain and discussed both the applied techniques and the results with experts.

We presented a technique revealing important details and patterns in motion data enabling the user to understand the observed motion patterns. Furthermore, it reduces over-plotting in by a combination of simplification and aggregation. Resulting from our expert study we plan to integrate further semantics into the visual representation. One important aspect coming up during the feedback session was an integration of land use data sets. Using land use categories (e.g., forest, agriculture, urban, etc.) the analyst understands the requirements of resources for specific movement behaviors. Another idea we want to follow is the temporal segmentation of the graph taking only time features as seasons or day-time) into account.

\section{ACKNOWLEDGMENTS}

This work has been partly funded by the German Research Society (DFG) under the grant GK-1042, Explorative Analysis and Visualization of Large Information Spaces, Konstanz and the SPP 1335, sub-project Visual Analysis of Movement and Event Data in Spatiotemporal Context. We thank Kamran Safi and Bart Kranstauber for the many rounds of feedback and for sharing their tracking data. Furthermore, we would like to express our gratitude to Bruce Patterson from the Field Museum and Roland Kays for the permission to use their data and for their help in the interpretation.

\section{REFERENCES}

[1] Douglas, D. and Peucker, T., "Algorithms for the reduction of the number of points required to represent a digitized line or its caricature," Cartographica: The International Journal for Geographic Information and Geovisualization 10(2), 112-122 (1973).

[2] Nakos, B. and Miropoulos, V., "Local length ratio as a measure of critical points detection for line simplification," in [Fifth Workshop on Progress in Automated Map Generalization, Paris, France], (2003).

[3] Burghardt, D., "Controlled line smoothing by snakes," GeoInformatica 9(3), 237-252 (2005).

[4] Agarwal, P. K., Har-Peled, S., Mustafa, N. H., and Wang, Y., "Near-linear time approximation algorithms for curve simplification," Algorithmica 42, 203-219 (2005).

[5] Laube, P. and Purves, R., "Cross-scale movement trajectory analysis," in [GIS Research UK 18th Annual Conference GISRUK], 103-107 (2010).

[6] Ellis, G. and Dix, A., "Density control through random sampling: an architectural perspective," in [Information Visualisation, 2002. Proceedings. Sixth International Conference on ], 82-90, IEEE (2002).

[7] Dykes, J. and Mountain, D., "Seeking structure in records of spatio-temporal behaviour: visualization issues, efforts and applications," Computational Statistics \& Data Analysis 43(4), 581-603 (2003).

[8] Forer, P. and Huisman, O., "Space, Time and Sequencing: Substitution at the Physical/Virtual Interface," Information, Place, and Cyberspace: Issues in Accessibility, 73 (2000).

[9] Mountain, D., "Visualizing, Querying and Summarizing Individual Spatio-Temporal Behaviour," Exploring Geovisualization, 181-200 (2005).

[10] Willems, N., Van De Wetering, H., and Van Wijk, J., "Visualization of vessel movements," Computer Graphics Forum 28(3), 959-966 (2009). 
[11] Scheepens, R., Willems, N., van de Wetering, H., and van Wijk, J. J., "Interactive visualization of multivariate trajectory data with density maps," in [Pacific Visualization Symposium (PacificVis), 2011 IEEE], 147-154, IEEE (2011).

[12] Scheepens, R., Willems, N., van de Wetering, H., Andrienko, G., Andrienko, N., and van Wijk, J. J., "Composite density maps for multivariate trajectories," Visualization and Computer Graphics, IEEE Transactions on 17(12), 2518-2527 (2011).

[13] Guo, D., "Flow Mapping and Multivariate Visualization of Large Spatial Interaction Data," IEEE Transactions on Visualization and Computer Graphics 15, 1041-1048 (October 2009).

[14] Phan, D., Xiao, L., Yeh, R., Hanrahan, P., and Winograd, T., "Flow Map Layout," in [Proceedings of the IEEE Symposium on Information Visualization ], 219-224 (2005).

[15] Tobler, W., "Experiments in migration mapping by computer," The American Cartographer 14(2), 155-163 (1987).

[16] Boyandin, I., Bertini, E., Bak, P., and Lalanne, D., "Flowstrates: An approach for visual exploration of temporal origin-destination data," in [Computer Graphics Forum ], 30(3), 971-980, Wiley Online Library (2011).

[17] Wood, J. and Dykes, J., "Spatially Ordered Treemaps," IEEE Trans. on Visualization and Computer Graphics 14(6), 1348-1355 (2008).

[18] Andrienko, G. and Andrienko, N., "Spatio-temporal aggregation for visual analysis of movements," in [Visual Analytics Science and Technology, 2008. VAST'08. IEEE Symposium on ], 51-58, IEEE (2008).

[19] Andrienko, G. and Andrienko, N., "A general framework for using aggregation in visual exploration of movement data," The Cartographic Journal 47(1), 22-40 (2010).

[20] Andrienko, G., Andrienko, N., Burch, M., and Weiskopf, D., "Visual analytics methodology for eye movement studies," Visualization and Computer Graphics, IEEE Transactions on 18(12), 2889-2898 (2012).

[21] Li, X., Çöltekin, A., and Kraak, M.-J., "Visual exploration of eye movement data using the space-time-cube," in [Geographic Information Science], 295-309, Springer (2010).

[22] Grundy, E., Jones, M. W., Laramee, R. S., Wilson, R. P., and Shepard, E. L., "Visualisation of sensor data from animal movement," in [Computer Graphics Forum], 28(3), 815-822, Wiley Online Library (2009).

[23] Buchin, M., Driemel, A., van Kreveld, M. J., and Sacristan, V., "An algorithmic framework for segmenting trajectories based on spatio-temporal criteria," in [GIS], 202-211 (2010).

[24] Anagnostopoulos, A., Vlachos, M., Hadjieleftheriou, M., Keogh, E., and Yu, P., "Global distance-based segmentation of trajectories," in [Proceedings of 12th ACM SIGKDD], 34-43 (2006).

[25] Guerrero, J., Garcia, J., and Molina, J., "Air traffic trajectories segmentation based on time-series sensor data," Sensor Fusion and its Applications (2010).

[26] Helmuth, J., Burckhardt, C., Koumoutsakos, P., Greber, U., and Sbalzarini, I., "A novel supervised trajectory segmentation algorithm identifies distinct types of human adenovirus motion in host cells," Journal of structural biology 159(3), 347-358 (2007).

[27] Panagiotakis, C., Pelekis, N., Kopanakis, I., Ramasso, E., and Theodoridis, Y., "Segmentation and Sampling of Moving Object Trajectories based on Representativeness," IEEE Transactions on Knowledge and Data Engineering (2011).

[28] Laube, P., Imfeld, S., and Weibel, R., "Discovering relative motion patterns in groups of moving point objects," International Journal Geographic Information Science 19(6), 639-668 (2005).

[29] Dodge, S., Weibel, R., and Forootan, E., "Revealing the physics of movement: Comparing the similarity of movement characteristics of different types of moving objects," Computers, Environment and Urban Systems 33(6), 419-434 (2009).

[30] Ester, M., Kriegel, H., Sander, J., and Xu, X., "A density-based algorithm for discovering clusters in large spatial databases with noise," in [Proceedings of the 2nd International Conference on Knowledge Discovery and Data mining ], 1996, 226-231, Portland: AAAI Press (1996).

[31] Plaisant, C., Carr, D., and Shneiderman, B., "Image-Browser Taxonomy and Guidelines for Designers," IEEE Softw. 12, 21-32 (Mar. 1995).

[32] Tufte, E., [Envisioning information], no. Bd. 914 in Envisioning Information, Graphics Press (1990). 